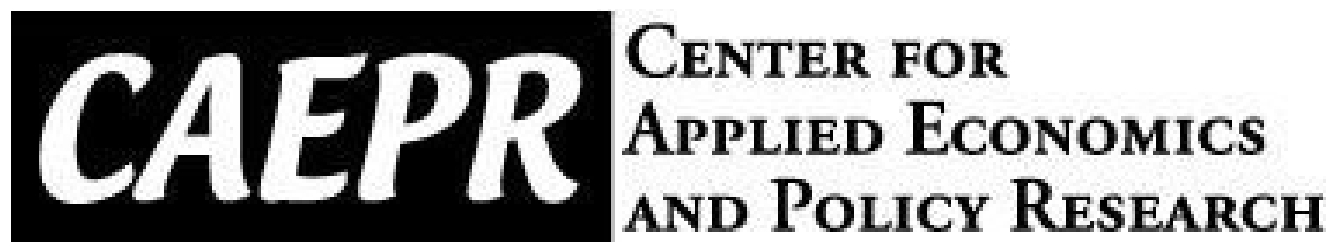

CAEPR Working Paper

\#2015-023

\title{
Uniformly Consistent Estimation of Linear Regression Models with Strictly Exogenous Instruments
}

\section{Juan Carlos Escanciano Indiana University}

January 31, 2016

This paper can be downloaded without charge from the Social Science Research Network electronic library at http://papers.ssrn.com/sol3/papers.cfm?abstract id=2706577

The Center for Applied Economics and Policy Research resides in the Department of Economics at Indiana University Bloomington. CAEPR can be found on the Internet at:

http://www.indiana.edu/ caepr. CAEPR can be reached via email at caepr@indiana.edu or via phone at 812-855-4050.

(C)2015 by Juan Carlos Escanciano. All rights reserved. Short sections of text, not to exceed two paragraphs, may be quoted without explicit permission provided that full credit, including (C) notice, is given to the source. 


\title{
Uniformly Consistent Estimation of Linear Regression Models with Strictly Exogenous Instruments*
}

\author{
Juan Carlos Escanciano ${ }^{\dagger}$ \\ Indiana University
}

January 31, 2016

\begin{abstract}
This paper investigates estimation of linear regression models with strictly exogenous instruments under minimal identifying assumptions. Under this setting the commonly used Instrumental Variables (IV) estimators are not uniformly consistent (uniformity is in the underlying data generating process). This negative result is due to the lack of "continuity" in the identification of IV caused by weak instruments. This paper introduces a uniformly consistent estimator in this setting. The proposed estimator, called the Integrated Instrumental Variables (IIV) estimator, is a weighted least squares estimator with trivial implementation. Monte Carlo evidence supports the theoretical claims and suggests that the IIV estimator is a robust alternative to IV and optimal IV in finite samples under weak identification and strictly exogenous instruments. In an application with quarterly UK data the IIV estimates a positive and significant elasticity of intertemporal substitution and an equally sensible estimate for its reciprocal, in contrast to IV methods that fail to identify these parameters.
\end{abstract}

Keywords: Identification; Instrumental variables; Weak instruments; Efficient IV; Intertemporal elasticity of substitution.

JEL classification: C13, C26.

${ }^{*}$ I thank Lin Zhu for excellent research assistance, and Don Andrews, Marine Carrasco, Ed Vytlacil and Jeff Wooldridge for useful comments. A previous version of this paper was circulated under the title: "The Integrated Instrumental Variables Estimator: Exploiting Nonlinearities for Identification of Linear Models", dated February 2, 2010.

${ }^{\dagger}$ Department of Economics, Indiana University, 105 Wylie Hall, 100 S. Woodlawn Avenue, Bloomington, IN 47405-7104, USA. E-mail: jescanci@indiana.edu. 


\section{Introduction}

The linear regression model continues to be the workhorse model in applied research. In the presence of endogenous regressors these models are often estimated by Instrumental Variables (IV) methods, where "exogeneity" and "relevance" conditions are required for the validity of the resulting inferences. The recent literature on weak instruments has emphasized the practical importance of uniform inferences (uniformity is in the underlying probability generating the data, see, e.g., Staiger and Stock, 1997, and Dufour, 1997). The lack of uniformly consistent estimators in the standard IV setting, caused by the possibility of weak instruments, has motivated a focus on testing and confidence intervals (see Stock, Wright and Yogo, 2002, and Andrews and Stock, 2005, for recent surveys of the literature). This paper departs from this literature by investigating uniform estimation in a setting with strictly exogenous instruments. First, it establishes identification under minimal assumptions (i.e. necessary and sufficient conditions for identification). It then shows that commonly used IV estimators are not uniformly consistent under these assumptions. Then, the paper proceeds by proposing an estimator, called the Integrated Instrumental Variables (IIV) estimator, that is uniformly consistent under nearly the minimal identifying assumptions. Monte Carlo simulations and an empirical application to estimating the elasticity of intertemporal substitution show that the uniform consistency property translates into excellent finite sample behaviour for the IIV estimator, comparing favorably with IV and optimal IV estimators. The theoretical and empirical evidence, together with the simplicity of implementation, make the IIV estimator a robust alternative to commonly used IV estimators with strictly exogenous instruments.

The data $\left\{W_{i} \equiv\left(Y_{i}, X_{i}^{\prime}, Z_{i}^{\prime}\right)^{\prime}\right\}$ is a sequence of independent and identically distributed (iid) observations, having the same distribution as $W \equiv\left(Y, X^{\prime}, Z^{\prime}\right)^{\prime}$, defined on the probability space $(\Omega, \mathcal{F}, P)$, and taking values in $\mathbb{R} \times \mathbb{R}^{p} \times \mathbb{R}^{k}, p, k \in \mathbb{N}$. Henceforth, $A^{c}, A^{\prime}$ and $|A|$ denote the complex conjugate, the matrix transpose and the Euclidean norm of $A,|A|=\left(\operatorname{tr}\left(A^{c} A\right)\right)^{1 / 2}$, respectively. The components of $W$ are such that $E[|Y|]<\infty$, $E\left[|X|^{2}\right]<\infty$,

$$
Y=X^{\prime} \theta_{0}+\varepsilon,
$$

where $\theta_{0} \in \mathbb{R}^{p}$, and $Z$ is a vector of strictly exogenous instruments, i.e.

$$
E[\varepsilon \mid Z]=0 \text { almost surely (a.s.). }
$$

This paper deals with identification and estimation of the model defined in (1)-(2).

The linearity in (1) is often justified on the basis of simplicity and has been shown to be a useful approximation in many economic applications. Economic theory or randomization 
can be used to justify (2). For example, in dynamic Euler equations the strict exogeneity naturally holds provided $Z$ is in the agent's information set.

We now introduce the minimal identifying assumption for $\theta_{0}$ in (1)-(2). We call this assumption the Nonparametric Relevance (NR) assumption, emphasizing the use of nonparametric regressions, as opposed to more traditional rank conditions in IV that use parametric linear regressions. Define the function $m(z):=E[X \mid Z=z]$ and the random variable $\tilde{Z}=m(Z)$ (henceforth, we drop a.s. for simplicity of exposition). Taking conditional means in (1) with respect to $Z$, we obtain $E[Y \mid Z]=\tilde{Z}^{\prime} \theta_{0}$, and hence

$$
E[Y \mid \tilde{Z}]=\tilde{Z}^{\prime} \theta_{0}
$$

This equation shows $\theta_{0}$ is identified under the following condition.

Assumption NR: $E\left[\tilde{Z} \tilde{Z}^{\prime}\right]$ is positive definite (p.d).

Assumption NR is a nonparametric generalization of the classical rank condition. This identifying assumption is testable; see our empirical application for nonparametric tests for Assumption NR in the context of linearized Euler equations. Related conditions to Assumption NR have been used in the econometrics literature for nonparametric identification of certain models, see e.g. Das (2005) and Cai et al. (2006), but its use in linear models has not been emphasized to the best of our knowledge. The first result establishes the minimality of this assumption for identification. Its proof is standard and therefore omitted.

Proposition 1: In (1)-(2), $\theta_{0}$ is identified if and only if Assumption NR holds.

It follows from Proposition 1 that Assumption NR is implied by the traditional rank condition $\operatorname{rank}\left(E\left[Z X^{\prime}\right]\right)=p$ (and $\operatorname{rank}\left(E\left[Z Z^{\prime}\right]\right)=k$ ), which is usually assumed for identification with classical IV methods. ${ }^{1}$ We stress that Assumption NR allows for the possibility of having less instruments than endogenous variables, $k<p$, by exploiting nonlinearities in the dependence between $X$ and $Z$. In contrast, in the classical linear identification assumption the order condition $k \geq p$ is necessary. Thus, from this discussion we conclude that Classical IV requires the weaker exogeneity condition $E[\varepsilon Z]=0$, but this relaxation is at the cost of a stronger relevance condition that would be otherwise required under strict exogeneity. This tradeoff is most relevant for weak instruments, for which the classical rank condition fails, or it is closed to fail, but Assumption NR may hold. Our application to estimating the elasticity of intertemporal substitution with UK data seems to be such a case. Nevertheless,

\footnotetext{
${ }^{1}$ Given that Assumption NR is sufficient and necessary for identification, we refer to it as the minimal identifying assumption in the rest of the paper.
} 
our methods may outperform classical IV methods also with strong instruments, as seen in the Monte Carlo Section below.

Reliable inference requires uniformity in the underlying probability measure $P$ over a large class of probabilities. ${ }^{2}$ Henceforth, we assume $P \in \mathcal{P}$, where $\mathcal{P}$ is the class of probabilities satisfying the assumptions above, i.e.

$$
\mathcal{P}:=\left\{\begin{array}{c}
P: E_{P}\left[Y-X^{\prime} \theta_{0} \mid Z\right]=0, E_{P}[|Y|] \leq C, E_{P}\left[|X|^{2}\right] \leq C \\
\text { and } \lambda_{\min }\left(E_{P}\left[m_{P}(Z) m_{P}^{\prime}(Z)\right]\right) \geq C
\end{array}\right\}
$$

where, henceforth, the dependence on $P$ of the expectations will be emphasized (e.g. in $\left.m_{P}(Z)=E_{P}[X \mid Z]\right), \lambda_{\min }(A)$ denotes the minimum eigenvalue of a symmetric matrix $A$, and $C$ is a generic positive constant that may change from expression to expression. The independence of $C$ on $P$ will be useful in proving uniformity results. The condition $\lambda_{\min }\left(E_{P}\left[m_{P}(Z) m_{P}^{\prime}(Z)\right]\right) \geq C$ is slightly stronger than Assumption NR, as it assumes that the minimum eigenvalue is uniformly bounded away from zero. ${ }^{3}$

Let $\hat{\theta}_{I V}$ denote the Two-Stage Least Squares (TSLS) estimator (assuming it exists). The following result shows that this commonly used estimator is not uniformly consistent over $\mathcal{P}$. Its proof is simple: the TSLS estimator fails to identify $\theta_{0}$ over $\mathcal{P}$, i.e. the mapping

$$
P \rightarrow \theta_{I V}(P):=\left(E_{P}\left[X Z^{\prime}\right]\left(E_{P}\left[Z Z^{\prime}\right]\right)^{-1} E_{P}\left[Z X^{\prime}\right]\right)^{-1} E_{P}\left[X Z^{\prime}\right]\left(E_{P}\left[Z Z^{\prime}\right]\right)^{-1} E_{P}[Z Y]
$$

that the TSLS is estimating when $W$ is generated from $P$ fails to exists for all $P \in \mathcal{P}$. ${ }^{4}$

Proposition 2: TSLS is not uniformly consistent over $\mathcal{P}$, i.e. for all $\varepsilon>0$,

$$
\sup _{P \in \mathcal{P}} P\left(\left|\hat{\theta}_{I V}-\theta_{0}\right|>\varepsilon\right) \nrightarrow 0
$$

This result holds even if we include in $\mathcal{P}$ the classical rank condition $\operatorname{rank}\left(E_{P}\left[Z X^{\prime}\right]\right)=p$, as there are weakly converging sequences of probabilities in $\mathcal{P}$ satisfying the rank condition for all elements of the sequence but for its limit (see, e.g. Staiger and Stock, 1997). That is, IV fails to identify $\theta_{0}$ "uniformly over $\mathcal{P}$ ". See Dufour (1997) for related discussion.

This paper introduces an estimator that is uniformly consistent over $\mathcal{P}$. The new Inte-

\footnotetext{
${ }^{2}$ Of course, as noted by Bahadur and Savage (1956) the class cannot be too large. With uniformity over a class $\mathcal{P}$ the sample size required to achieve "good" approximations does not depend on $P \in \mathcal{P}$.

${ }^{3}$ We refer to this slightly stronger condition as the "nearly minimal identifying assumption".

${ }^{4}$ Identification in $\mathcal{P}$ is referred to as "uniform identification" with respect to $\mathcal{P}$ in Manski (1988, p.4). In this paper, we refer to "uniform identification over $\mathcal{P}$ " as the following stronger condition: identification holds on the closure of $\mathcal{P}$ (with respect to the topology of weak convergence). Since our $\mathcal{P}$ is closed both concepts coincide in our setting.
} 
grated Instrumental Variables (IIV) estimator for $\theta_{0}$ is a weighted least squares estimator, computed as

$$
\hat{\theta}_{I I V}:=\left(\mathbf{X}^{\prime} \Omega \mathbf{X}\right)^{-1}\left(\mathbf{X}^{\prime} \Omega \mathbf{Y}\right)
$$

where $\mathbf{X}$ is the $n \times p$ design matrix with rows $X_{i}^{\prime}, \mathbf{Y}=\left(Y_{1}, \ldots, Y_{n}\right)^{\prime}, \Omega$ is the $n \times n$ matrix with elements $\exp \left(-0.5\left|Z_{i}-Z_{s}\right|^{2}\right), 1 \leq i, s \leq n$, and $n \geq 1$ is the sample size. Section 2 provides motivation for this choice of weighting matrix $\Omega$, while Section 3 shows $\sqrt{n}\left(\hat{\theta}_{I I V}-\theta_{0}\right)$ is asymptotically normal with an asymptotic variance that is consistently estimated by $\widehat{\Gamma}=$ $\widehat{\Sigma}^{-1} \widehat{\Lambda} \widehat{\Sigma}^{-1}$, where $\widehat{\Sigma}=\left(\mathbf{X}^{\prime} \Omega \mathbf{X}\right) / n^{2}, \widehat{\Lambda}=\left(\mathbf{X}^{\prime} \Omega D \Omega \mathbf{X}\right) / n^{3}$ and $D$ is a diagonal matrix with components $\left\{\widehat{\varepsilon}_{i}^{2}\right\}_{i=1}^{n}$, where $\widehat{\varepsilon}_{i}=Y_{i}-X_{i}^{\prime} \hat{\theta}_{I I V}$ are IIV residuals.

An estimator that provides identification of $\theta_{0}$ over the whole $\mathcal{P}$ is the optimal IV estimator (cf. Amemiya, 1974, 1977, Robinson, 1976, Chamberlain 1982, Newey 1990). This IV estimator uses the optimal instruments $Z^{*}:=\sigma_{P}^{-2}(Z) m_{P}(Z)$, where $\sigma_{P}^{2}(Z):=E_{P}\left[\varepsilon^{2} \mid Z\right]$. However, this estimator also fails to be uniformly consistent over $\mathcal{P}$. This failure follows from $\sigma_{P}^{2}(Z)$ being either too close to zero or to infinity. See also the general results in Pötscher (2002), who showed the lack of uniformly consistent estimators of $m_{P}(Z)$ and $\sigma_{P}^{2}(Z)$ over $\mathcal{P}$. These results are independent of the estimators used for $m_{P}(Z)$ and $\sigma_{P}^{2}(Z)$, and apply equally to estimators that are asymptotically equivalent to the optimal ones, such as that proposed in Carrasco (2012). The Monte Carlo simulations below provide empirical evidence of the lack of uniform consistency for optimal IV estimators, and suggest that the new estimator significantly outperforms optimal IV estimates when the minimal identifying Assumption NR is closed to not be satisfied.

The new estimator belongs to the class of minimum distance estimators, and is similar in spirit to the estimator proposed by Dominguez and Lobato (2004). These authors pointed out that in nonlinear models, IV, or the more general Generalized Method of Moments, may fail to identify. This problem does not arise in our linear setting, as optimal IV identifies $\theta_{0}$ over $\mathcal{P}$. The distinctive feature of our paper is the emphasis on uniformity (in identification, consistency and asymptotic normality). There is previous research in econometrics on uniform inference, but this work has mainly focussed on uniformly valid confidence sets and tests, and different models. ${ }^{5}$ Kasy (2015) has recently investigated uniformity in the Delta Method, and has shown that the IV estimator fails to be uniformly asymptotically normal in the classical IV setting. The present paper uncovers a tradeoff between the exogeneity and relevance condition and shows its potential utility in the presence of weak instruments through a new uniformly consistent and asymptotically normal estimator.

\footnotetext{
${ }^{5}$ See, e.g., Imbens and Manski (2004), Leeb and Pötscher (2005), Mikusheva (2007), Andrews and Guggenberger (2009) and Belloni, Chernozhukov, Fernandez-Val and Hansen (2015).
} 
The rest of the paper is organized as follows. Section 2 introduces formally the IIV estimator, and shows that this estimator identifies $\theta_{0}$ over the whole $\mathcal{P}$. In Section 3 we establish the uniform consistency and uniform asymptotic normality for the IIV estimator. Readers not interested in the technical underpinnings can skip Sections 2 and 3. Section 4 reports the results of some Monte Carlo experiments showing that the proposed estimator performs very well in finite samples and compares favorably with IV estimators, including optimal ones. Section 5 contains an application to estimating the elasticity of intertemporal substitution for international data. Section 6 concludes and discusses future research. An Appendix gathers the mathematical proofs of our main results.

\section{The Integrated Instrumental Variables Estimator}

This section introduces the IIV estimator. To that end, define

$$
h_{y, P}(v):=E_{P}\left[Y \exp \left(i v^{\prime} Z\right)\right], \quad v \in \mathbb{R}^{k},
$$

where $i=\sqrt{-1}$. The function $h_{y, P}$ characterizes the reduced form regression $E_{P}[Y \mid Z]$. Likewise, define $h_{x, P}(v):=E_{P}\left[X \exp \left(i v^{\prime} Z\right)\right]$ and $h_{\varepsilon, P}(v):=E_{P}\left[\varepsilon \exp \left(i v^{\prime} Z\right)\right]$. Note that (2) implies $h_{\varepsilon, P}(\cdot) \equiv 0$. Following Bierens (1982), the reciprocal is also true, $h_{\varepsilon, P}(\cdot) \equiv 0$ implies the strict exogeneity condition (2).

Since $h_{y, P}(v)$ is a linear transformation in $Y$, we obtain

$$
h_{y, P}^{c}(v)=h_{x, P}^{c}(v) \theta_{0},
$$

Multiplying both sides of $(5)$ by $h_{x, P}(v)$, evaluating $v$ at $v=V$, with $V$ a standard Gaussian random vector in $\mathbb{R}^{k}$, and taking expectations with respect to $V$ we get

$$
E_{V}\left[h_{x, P}(V) h_{y, P}^{c}(V)\right]=E_{V}\left[h_{x, P}(V) h_{x, P}^{c}(V)\right] \theta_{0}
$$

Note that $E_{V}\left[h_{x, P}(V) h_{x, P}^{c}(V)\right]$ and $E_{V}\left[h_{x, P}(V) h_{y, P}^{c}(V)\right]$ exist for $P \in \mathcal{P}$. Moreover, we have the following result, which is proved in the Appendix.

Proposition 3: Assumption NR is equivalent to

$$
E_{V}\left[h_{x, P}(V) h_{x, P}^{c}(V)\right] \text { is p.d. }
$$


In view of Proposition 3, under Assumption NR $\theta_{0}$ is identified over the whole $\mathcal{P}$ as

$$
\theta_{0} \equiv \theta_{0}(P)=\left(E_{V}\left[h_{x, P}(V) h_{x, P}^{c}(V)\right]\right)^{-1} E_{V}\left[h_{x, P}(V) h_{y, P}^{c}(V)\right]
$$

This identification result and the analog principle suggest the IIV estimator

$$
\hat{\theta}_{I I V}:=\theta_{0}\left(P_{n}\right)=\left(E_{V}\left[h_{x, P_{n}}(V) h_{x, P_{n}}^{c}(V)\right]\right)^{-1} E_{V}\left[h_{x, P_{n}}(V) h_{y, P_{n}}^{c}(V)\right]
$$

where $P_{n}$ is the empirical probability measure associated to $\left\{W_{i} \equiv\left(Y_{i}, X_{i}^{\prime}, Z_{i}^{\prime}\right)^{\prime}\right\}_{i=1}^{n}$. That is, $h_{y, P_{n}}(v):=n^{-1} \sum_{s=1}^{n} Y_{s} \exp \left(i v^{\prime} Z_{s}\right)$ and $h_{x, P_{n}}(v):=n^{-1} \sum_{s=1}^{n} X_{s} \exp \left(i v^{\prime} Z_{s}\right)$. After some simple algebra it can be shown that $\hat{\theta}_{I I V}$ can be computed as $(3)$.

Following the logic of Bierens (1982) and Stinchcombe and White (1998), other estimators different from $\hat{\theta}_{I I V}$ can be constructed with similar uniform consistent properties if we replace quantities such as $E_{P}\left[Y \exp \left(i v^{\prime} Z\right)\right]$ by $E_{P}[Y w(Z, v)]$, for other suitable choices of $w(Z, v)$. For instance, if we use $w(Z, v)=1(Z \leq v)$, where $Z \leq v$ is understood coordinate-wise, we get an estimator in the spirit of that proposed by Dominguez and Lobato (2004) in a more general set-up. Note the indicator $1(Z \leq v)$ may lead to misleading inferences in applications where we have a large number of instruments, i.e. large $k$, as many $1\left(Z_{i} \leq v\right)$ could be zero when $v$ is evaluated at the sample observations; see e.g. Escanciano (2006) for further discussion and empirical evidence. The choice $\exp \left(i v^{\prime} Z_{t}\right)$ leads to a computationally simple estimator that is robust to moderate and large values of the number of instruments $k$.

\section{Uniform Asymptotic Theory for the IIV Estimator}

This section establishes uniform asymptotic theory for $\hat{\theta}_{I I V} \cdot{ }^{6}$ To that end, it is convenient to use asymptotic theory for random elements taking values in a suitable Hilbert space; see van der Vaart and Wellner (1996, Chapter 1.8). We need some basic notation. Let $L_{2}(\phi)$ be the Hilbert space of all complex-valued and square $\phi$-integrable functions, where $\phi$ is the $k$-dimensional multivariate standard Gaussian density. In $L_{2}(\phi)$ we define the inner product

$$
\langle f, g\rangle:=\int_{\mathbb{R}^{k}} f(v) g^{c}(v) \phi(v) d v .
$$

\footnotetext{
${ }^{6}$ Uniform convergence in distribution means the following: $T_{n} \rightarrow_{d} T$ uniformly over $\mathcal{P}$ iff $\sup _{P \in \mathcal{P}} \mid E_{P}\left[f\left(T_{n}\right)-E_{P}[f(T)] \mid \rightarrow 0\right.$ for all bounded and continuous functions $f(\cdot)$.
} 
$L_{2}(\phi)$ is endowed with the natural Borel $\sigma$-field induced by the norm $\|\cdot\|=\langle\cdot, \cdot\rangle^{1 / 2}$. We denote by $\stackrel{L_{2}}{\longrightarrow}$ convergence in probability in $L_{2}(\phi)$, i.e., $h_{n} \stackrel{L_{2}}{\longrightarrow} h \Longleftrightarrow\left\|h_{n}-h\right\| \stackrel{P}{\longrightarrow} 0$. We shall use uniform in $\mathcal{P}$ versions of the $\stackrel{L_{2}}{\longrightarrow}$ convergence.

Using the introduced notation, we can write

$$
\hat{\theta}_{I I V}=\left\langle h_{x, P_{n}}, h_{x, P_{n}}\right\rangle^{-1}\left\langle h_{x, P_{n}}, h_{y, P_{n}}\right\rangle
$$

where, with some abuse of notation, we have denoted by $\left\langle h_{x, P_{n}}, h_{x, P_{n}}\right\rangle$ the $p \times p$ matrix with elements $\left\langle h_{x_{j}, P_{n}}, h_{x_{k}, P_{n}}\right\rangle$, where $h_{x_{j}, P_{n}}$ is the $j-t h$ component of $h_{x, P_{n}}$. Now, uniform consistency of $\hat{\theta}_{I I V}$ will follow from the uniform law of large numbers in $L_{2}(\phi)$ for $h_{x, P_{n}}$ and $h_{y, P_{n}}$, i.e. from $h_{x, P_{n}} \stackrel{L_{2}}{\longrightarrow} h_{x, P}$ and $h_{y, P_{n}} \stackrel{L_{2}}{\longrightarrow} h_{y, P}$, uniformly in $P \in \mathcal{P}$, and the continuity of the inner product.

Theorem 1: The IIV estimator is uniformly consistent over $\mathcal{P}$, i.e. for all $\varepsilon>0$,

$$
\sup _{P \in \mathcal{P}} P\left(\left|\hat{\theta}_{I I V}-\theta_{0}\right|>\varepsilon\right) \longrightarrow 0
$$

An implication of uniform consistency is that the mapping that IIV is identifying, i.e $P \rightarrow$ $\theta_{0}(P)$ in (8), is continuous over $\mathcal{P}$ (with the variational distance); see Proposition 1.A in Bickel et al. (1993).

The arguments of Theorem 1 and some further results proved below show that, uniformly in $P \in \mathcal{P}$,

$$
\sqrt{n}\left(\hat{\theta}_{I I V}-\theta_{0}\right)=\left\langle h_{x, P}, h_{x, P}\right\rangle^{-1} \sqrt{n}\left\langle h_{x, P}, h_{\varepsilon, P_{n}}\right\rangle+o_{P}(1) .
$$

The term $\sqrt{n}\left\langle h_{x, P}, h_{\varepsilon, P_{n}}\right\rangle$ is simply a standardized sample mean, i.e.

$$
\sqrt{n}\left\langle h_{x, P}, h_{\varepsilon, P_{n}}\right\rangle=\frac{1}{\sqrt{n}} \sum_{i=1}^{n} \varepsilon_{i} H_{x, P}\left(Z_{i}\right)
$$

where $H_{x, P}(v):=E_{P}\left[X \exp \left(-0.5|v-Z|^{2}\right)\right]$.

For uniform asymptotic normality we need to slightly restrict the class of probabilities to

$$
\mathcal{P}^{A N}=\mathcal{P} \cap\left\{P: E_{P}\left[\varepsilon^{2}\right] \leq C\right\} .
$$

Then, the expansion (11) and the uniform Central Limit Theorem (CLT) given in Petrov $\left(1975\right.$, p. 118) yield the uniform asymptotic normality of $\sqrt{n}\left(\hat{\theta}_{I I V}-\theta_{0}\right)$ in the following result. Define $\Sigma_{P}:=\left\langle h_{x, P}, h_{x, P}\right\rangle, \Lambda_{P}:=E_{P}\left[\varepsilon^{2} H_{x}(Z) H_{x}^{\prime}(Z)\right]$ and $\Gamma_{P}:=\Sigma_{P}^{-1} \Lambda_{P} \Sigma_{P}^{-1}$. 
THEOREM 2: The IIV estimator is uniformly asymptotically normal in $\mathcal{P}^{A N}$, i.e

$$
\sqrt{n}\left(\hat{\theta}_{I I V}-\theta_{0}\right) \rightarrow_{d} N\left(0, \Gamma_{P}\right) \text { uniformly in } \mathcal{P}^{A N}
$$

Note that $\Sigma_{P}$ is non-singular uniformly in $\mathcal{P}^{A N}$ (cf. Proposition 1). The estimation of the asymptotic variance $\Gamma_{P}$ was discussed in the Introduction.

\section{Monte Carlo Simulations}

This section investigates the finite sample performance of the proposed estimator in comparison with IV methods in two simulation experiments. Specifically, we compare the performance of our IIV estimator $\left(\hat{\theta}_{I I V}\right)$, the standard IV estimator $\left(\hat{\theta}_{I V}\right)$ and the optimal IV (OIV) estimator $\left(\hat{\theta}_{O I V}\right)$.

In the first experiment we generate data $Y, X$ and $Z$ according to the model

$$
D G P 1:\left\{\begin{array}{l}
Y=\theta_{0} X+\varepsilon \\
X=\gamma Z^{2}+u
\end{array}, \quad\left(\begin{array}{l}
\varepsilon \\
u
\end{array}\right) \sim N\left(\left(\begin{array}{l}
0 \\
0
\end{array}\right),\left(\begin{array}{ll}
1 & \rho \\
\rho & 1
\end{array}\right)\right)\right.
$$

with $Z \sim N(\mu, 1)$ drawn independently of $\varepsilon$ and $u$. The true parameter is zero, i.e. $\theta_{0}=$ 0 . We consider several values for the parameters $(\mu, \gamma, \rho)$. Since $E_{P}[X Z]=\gamma E_{P}\left[Z^{3}\right]=$ $\gamma\left(\mu^{3}+3 \mu\right), \mu$ and $\gamma$ measure jointly the level of identification of the IV estimator using the instrument $Z$. Also, $\gamma$ alone measures the level of nonparametric identification, since $E_{P}\left[m^{2}(Z)\right]=\gamma^{2} E_{P}\left[Z^{4}\right]$. The correlation parameter $\rho$ measures the level of endogeneity. In the simulations we consider several combinations of the parameter values $\mu \in\{0,0.1,0.5,1\}$, $\gamma \in\{0.1,0.5,1\}$ and $\rho \in\{0.3,0.9\}$. In all the experiments we consider 1000 Monte Carlo replications.

The optimal estimator $\hat{\theta}_{O I V}$ requires nonparametric estimation of $m_{P}(Z)=E_{P}[X \mid Z]$ and $\sigma_{P}^{2}(Z)=E_{P}\left[\varepsilon^{2} \mid Z\right]$. We estimate these quantities with Nadaraya-Watson estimators using a Gaussian kernel and a bandwidth $h_{n}=1.06 \widehat{\sigma} n^{-1 / 3}$, which attains the optimal rate of convergence in the sense of minimizing the mean squared error of the resulting estimator, see Linton (2002). Here $\widehat{\sigma}$ is the sample standard deviation of $\left\{Z_{i}\right\}_{i=1}^{n}$. The term $1.06 \hat{\sigma}$ is not necessarily the optimal one, but we prefer to keep the exposition simple. We considered other bandwidth choices, but they led to qualitatively similar conclusions.

Table I reports the bias, standard error (SE), and root mean squared (RMS) error for the IV, OIV and IIV estimates for several values of $(\mu, \gamma, \rho)$ in DGP1 with $n=50$. It is evident from Table I that classical IV methods are very sensitive to the values of the parameters $(\mu, \gamma, \rho)$. In contrast, the proposed estimator has a satisfactory performance 
across the different parameter values (i.e. uniformly in the data generating processes). In particular, the results for the IIV estimator are not sensitive to the level of endogeneity $\rho$. For small and moderate values of $\mu$ and $\gamma$ the SE of IV and optimal IV are much higher than that of the proposed estimator. In particular, there are several cases where the RMS of our estimator is smaller than 100 times those of the optimal IV and IV estimators. The new estimator has in most cases lower biases than optimal IV and IV estimators. The RMS errors of the three estimators decrease with the level of nonparametric identification for a given level of endogeneity, that is, the larger $\gamma$ the lower the RMS, for a given value of $\rho$.

Tables I also illustrates the difference between the classical identification assumption and the nonparametric identification condition in Assumption NR. It is interesting to see the bad performance of the IV estimator when there is weak linear identification ( $\mu$ is small) but moderate or strong nonparametric identification ( $\gamma$ is large), as evidenced in the case $(\mu, \gamma, \rho)=(0.1,1,0.9)$. Note also how the optimal IV estimator is highly affected by the weak nonparametric identification, to the extent that the non-optimal IV leads in several cases to smaller RMS. For completeness, we also report at the bottom of Table I the case with no nonparametric identification $(\gamma=0)$. Again, the proposed estimator has the lowest RMS.

Table II contains the results for $n=200$. In general, we observe a reduction in biases and standard errors when nonparametric identification holds $(\gamma>0)$. But somewhat unexpectedly, there are cases where the SE of optimal IV increases as the sample size increases, even when $\gamma>0$, see for instance the case $(\mu, \gamma)=(0,0.1)$. An interesting feature of Table II is the low bias of the proposed estimator relative to IV methods.

In sum, these results show an omnibus out-performance of the IIV estimator over more traditional estimators, specially when identification is weak or moderate. 
TABLE I

Bias, Standard Error and Root Mean Square Error. N=50

\begin{tabular}{|c|c|c|c|c|c|c|c|c|c|c|c|}
\hline \multirow[b]{2}{*}{$\mu$} & \multirow[b]{2}{*}{$\gamma$} & \multirow[b]{2}{*}{$\rho$} & \multicolumn{3}{|c|}{ BIAS } & \multicolumn{3}{|c|}{$\mathrm{SE}$} & \multicolumn{3}{|c|}{ RMS } \\
\hline & & & $I V$ & $O I V$ & $I I V$ & $I V$ & $O I V$ & $I I V$ & $I V$ & $O I V$ & $I I V$ \\
\hline \multirow[t]{6}{*}{0} & 0.1 & 0.3 & -6.376 & 0.121 & 0.274 & 8.810 & 7.367 & .782 & 78.920 & 7.368 & 0.828 \\
\hline & & 0.9 & & .659 & 721 & & 0.698 & 439 & & 10.710 & .853 \\
\hline & 0.5 & 0.3 & -0.493 & -0.085 & 0.011 & 20.204 & 0.574 & 0.269 & 20.212 & 0.580 & 0.269 \\
\hline & & 0.9 & 0.666 & 141 & 0.070 & & 10.622 & 0.233 & & 10.623 & 0.244 \\
\hline & 1 & 0.3 & 0.098 & -0.004 & 0.004 & 4.539 & 0.127 & 0.136 & 4.5 & 0.127 & 0.136 \\
\hline & & 0.9 & 0.195 & -0.037 & 0.013 & 20.415 & 0.204 & 0.135 & 20.4 & 0.207 & 0.136 \\
\hline \multirow[t]{6}{*}{0.1} & 0.1 & 0.3 & -6.829 & -0.243 & 0.229 & 223.230 & 15.673 & 0.723 & 223. & 15.674 & 0.758 \\
\hline & & 0.9 & 0.542 & -2.526 & 0.712 & 19.377 & 101.543 & 0.414 & 19.3 & 101. & 0.824 \\
\hline & 0.5 & 0.3 & -1.026 & 0.096 & 0.033 & 37.674 & 3.212 & 0.260 & 37. & 3.213 & 0.262 \\
\hline & & 0.9 & -( & -0.143 & 0.06 & 2 & 66 & & & & 255 \\
\hline & 1 & 0.3 & 0.577 & -0.004 & 0.010 & 26.838 & 0.132 & 0.140 & 26.84 & 0.132 & 0.140 \\
\hline & & 0.9 & 1.746 & -0.045 & 0.006 & 41.650 & 0.242 & 0.126 & 41.66 & 0.2 & 0.126 \\
\hline \multirow[t]{6}{*}{0.5} & 0.1 & 0.3 & & 0.252 & 0.21 & & & & & & 0.697 \\
\hline & & 0.9 & -0.616 & -3.202 & & 13.207 & 118.581 & & & 118.630 & 0.749 \\
\hline & 0.5 & 0.3 & -0.088 & -0.004 & 0.014 & 2.817 & 0.742 & 0.210 & 2.818 & 0.742 & 0.211 \\
\hline & & 0.9 & -0.093 & -0.071 & & & 0.366 & & & 0.373 & 0.201 \\
\hline & 1 & 0.3 & -0.012 & -0.003 & -0.000 & 0.223 & 0.102 & 0.102 & 0.2 & 0.102 & 0.102 \\
\hline & & 0.9 & -0.021 & -0.016 & 0.011 & 0.339 & 0.111 & 0.101 & 0.340 & 0.112 & 0.101 \\
\hline \multirow[t]{6}{*}{1} & 0.1 & 0.3 & -0.552 & -3.274 & & & 110.624 & & & 110.670 & 0.545 \\
\hline & & 0.9 & 0.169 & 2.213 & 0.320 & 13.435 & 31.377 & 0.475 & 13.4 & 31.455 & 0.573 \\
\hline & 0.5 & 0.3 & -0.003 & -0.010 & 0.003 & 0.111 & 0.175 & 0.125 & 0.111 & 0.176 & 0.125 \\
\hline & & 0.9 & -0.009 & -0.031 & & & & 0.127 & & 0.150 & 0.127 \\
\hline & 1 & 0.3 & -0.001 & -0.002 & 0.000 & 0.053 & 0.065 & 0.062 & & 0.065 & 0.062 \\
\hline & & 0.9 & -0.002 & -0.003 & & & & 0.061 & & 0.065 & 0.061 \\
\hline \multirow[t]{2}{*}{0} & 0 & 0.3 & 0.215 & -0.049 & & & 16.821 & 0.846 & & 16.821 & 0.892 \\
\hline & & 0.9 & -0.625 & 1.674 & 0.910 & 57.407 & 17.477 & 0.393 & 57.410 & 17.557 & 0.992 \\
\hline
\end{tabular}


TABLE II

Bias, Standard Error and Root Mean Square Error. N=200

\begin{tabular}{|c|c|c|c|c|c|c|c|c|c|c|c|}
\hline \multirow[b]{2}{*}{$\mu$} & \multirow[b]{2}{*}{$\gamma$} & \multirow[b]{2}{*}{$\rho$} & \multicolumn{3}{|c|}{ BIAS } & \multicolumn{3}{|c|}{$\mathrm{SE}$} & \multicolumn{3}{|c|}{ RMS } \\
\hline & & & $I V$ & $O I V$ & $I I V$ & $I V$ & OIV & $I I V$ & $I V$ & OIV & $I I V$ \\
\hline \multirow[t]{6}{*}{0} & 0.1 & 0.3 & -0.785 & -0.831 & 0.137 & 6.080 & 35.078 & 0.519 & 2.609 & 35.880 & 0.536 \\
\hline & & & 0.530 & -0.472 & 0.439 & 7.464 & 16.911 & .413 & 17.472 & 16.918 & 0.603 \\
\hline & 0.5 & 0.3 & 0.063 & -0.014 & -0.000 & 14.995 & 0.120 & 0.142 & & 0.121 & 0.142 \\
\hline & & 0.9 & -0.236 & -0.039 & 0.008 & 17.791 & 0.155 & 0.130 & 17.792 & 0.160 & 0.130 \\
\hline & 1 & 0. & 0.512 & 0.001 & 0.004 & 7.750 & 0.062 & 0.071 & 7.767 & 0.062 & 0.071 \\
\hline & & 0.9 & -0.187 & -0.008 & 0.001 & 8.526 & 0.070 & 0.068 & 8.528 & 0.071 & 0.068 \\
\hline \multirow[t]{6}{*}{0.1} & 0.1 & 0.3 & -0.038 & -0.070 & 0.165 & 14.205 & 6.538 & 0.528 & 14.205 & 6.539 & 0.554 \\
\hline & & 0.9 & 1.158 & -1.307 & 0.415 & 15.816 & 67.639 & 0.400 & 15.858 & 67.651 & 0.577 \\
\hline & 0.5 & 0.3 & -0.302 & -0.003 & 0.011 & 6.454 & 0.127 & 0.132 & 6.461 & 0.127 & 0.132 \\
\hline & & 0.9 & -0.03 & -0.016 & 0.014 & 9.226 & 0.135 & .128 & 9.226 & 0.136 & 0.128 \\
\hline & 1 & 0.3 & 0.337 & 0.000 & 0.001 & 14.414 & 0.061 & 0.071 & 14.418 & 0.061 & 0.071 \\
\hline & & 0.9 & .097 & -0.006 & 0.003 & 4.220 & 0.065 & 0.067 & 4.221 & 0.066 & 0.067 \\
\hline \multirow[t]{6}{*}{0.5} & 0.1 & 0.3 & -0.431 & 0.213 & & 6.924 & 7.055 & & & 7.058 & 0.430 \\
\hline & & 0.9 & 0.179 & 0.732 & 0.252 & 14.682 & 87.858 & 0.356 & 14.683 & 87.861 & 0.436 \\
\hline & 0.5 & 0.3 & -0 & .006 & & & & & & & 0.104 \\
\hline & & 0.9 & -0.013 & -0.019 & & & 0.116 & & & 0.118 & 0.099 \\
\hline & 1 & 0.3 & 0.002 & -0.004 & 0.001 & 0.051 & 0.064 & 0.048 & 0.051 & 0.064 & 0.048 \\
\hline & & 0.9 & -0.001 & -0.003 & 0.001 & 0.053 & 0.053 & 0.051 & 0.053 & 0.053 & 0.051 \\
\hline \multirow[t]{6}{*}{1} & 0.1 & 0.3 & -0.034 & 0.142 & 0.023 & 0.325 & 22.013 & 0.292 & 0.326 & 22.013 & 0.293 \\
\hline & & 0.9 & -0.078 & -0.191 & 0.045 & & 398 & & & 6.401 & 0.291 \\
\hline & 0.5 & 0.3 & -0.000 & -0.003 & 0.001 & 0.052 & 0.076 & 0.061 & 0.052 & 0.076 & 0.061 \\
\hline & & 0.9 & -0.003 & -0.005 & 0.000 & 0.052 & 0.092 & 0.061 & 0.052 & 0.092 & 0.061 \\
\hline & 1 & 0.3 & -0.001 & -0.001 & -0.001 & 0.025 & 0.036 & 0.030 & 0.025 & 0.036 & 0.030 \\
\hline & & 0.9 & -0.000 & 0.000 & 0.000 & 0.024 & 0.039 & 0.029 & 0.024 & 0.039 & 0.029 \\
\hline \multirow[t]{2}{*}{0} & 0 & 0.3 & 0.429 & -1.773 & & & 26.902 & & & 26.961 & 0.870 \\
\hline & & 0.9 & -0.572 & 0.349 & 0.903 & 57.482 & 12.834 & 0.395 & 57.485 & 12.839 & 0.986 \\
\hline
\end{tabular}


In the second experiment we consider a regression model with an endogenous binary variable. The same model was investigated in Newey (1990). We generate data $Y, X$ and $Z$ according to the model

$$
D G P 2:\left\{\begin{array}{l}
Y=\theta_{01}+X \theta_{02}+\varepsilon \\
X=1\left(\alpha_{01}+\alpha_{02} Z+u>0\right)
\end{array}, \quad\left(\begin{array}{l}
\varepsilon \\
u
\end{array}\right) \sim N\left(\left(\begin{array}{l}
0 \\
0
\end{array}\right),\left(\begin{array}{ll}
1 & \rho \\
\rho & 1
\end{array}\right)\right)\right.
$$

with $Z \sim N(0,1)$ independent of $\varepsilon$ and $u$. The true parameters are $\theta_{01}=\theta_{02}=1$. The parameter $\alpha_{01}$ is fixed at $\alpha_{01}=1$, but we consider different values for the parameters $\left(\alpha_{02}, \rho\right)$. Here, the parameters $\theta_{0}=\left(\theta_{01}, \theta_{02}\right)^{\prime}$ are nonparametrically identified if the propensity score $p(z)=P(X=1 \mid Z=z)$ is nonlinear in $z$. Since $p(z)=\Phi\left(\alpha_{01}+\alpha_{02} z\right)$, nonparametric identification (i.e. Assumption NR) holds provided $\alpha_{02} \neq 0$. Thus, $\left|\alpha_{02}\right|$ measures the level of nonparametric identification. The correlation parameter $\rho$ measures the level of endogeneity. In the simulations we consider several combinations of the parameter values $\alpha_{02} \in\{1,0.5,0.1\}$ and $\rho \in\{0.2,0.8\}$.

We compare the performance of our IIV estimator, the standard IV estimator using the instrument $Z$ for $X$, and the optimal IV estimator computed with a series estimator for the optimal instrument $p(Z)$. Note that no estimation of the conditional variance is considered here. We follow Newey (1990) and estimate $p(z)$ by $\hat{p}(z)=A^{q}(z)^{\prime} \gamma_{n}$, where $A^{q}(z)=\left(A_{1}(z), \ldots, A_{k}(z)\right), A_{j}(z)=z^{j-1}, j=1, \ldots, q, \gamma_{n}=\left[S_{n}^{\prime} S_{n}\right]^{-1} S_{n}^{\prime} \mathbf{Y}$,

$$
\mathbf{Y}:=\left[\begin{array}{c}
Y_{1} \\
\vdots \\
Y_{n}
\end{array}\right] \text { and } S_{n}:=\left[\begin{array}{c}
A^{q}\left(Z_{1}\right)^{\prime} \\
\vdots \\
A^{q}\left(Z_{n}\right)^{\prime}
\end{array}\right]
$$

We report results with $q=7$, but other values of $q$ led to similar conclusions. Similarly, other choices of basis functions gave comparable results. These unreported simulations can be obtained from the author upon request.

Table III reports results for estimates of $\theta_{01}$ and $\theta_{02}$ for a sample size of $n=100$. For all parameter values considered our estimator for $\theta_{01}$ presents a smaller RMS than IV and optimal IV, specially for low values of $\alpha_{02}$. It is remarkable the robustness of the new estimator to weak nonparametric identification when $\alpha_{02}=0.1$. For this case the RMS of the IV estimator is more than 50 times that of the IIV estimate. For $\theta_{02}$, the optimal IV has the smallest RMS for large values of $\alpha_{02}$, but its performance substantially deteriorates when $\alpha_{02}$ decreases, showing one more time that optimal IV is rather sensitive to weak nonparametric identification (it is not uniformly consistent). 


\section{TABLE III}

Bias, Standard Error and Root Mean Square Error. DGP2. $n=100$

\begin{tabular}{cccccccccccc}
\hline \hline & & \multicolumn{3}{c}{ BIAS } & \multicolumn{3}{c}{ SE } \\
\cline { 3 - 12 } & $\alpha_{02}$ & $\rho$ & $I V$ & $O I V$ & $I I V$ & $I V$ & $O I V$ & $I I V$ & $I V$ & $O I V$ & $I I V$ \\
\hline$\theta_{01}$ & 1 & 0.2 & 1.010 & 1.760 & 1.008 & 0.379 & 0.108 & 0.383 & 1.079 & 1.763 & 1.078 \\
& & 0.8 & 1.029 & 1.762 & 1.020 & 0.369 & 0.119 & 0.375 & 1.093 & 1.766 & 1.087 \\
& 0.5 & 0.2 & 1.082 & 1.813 & 1.034 & 1.004 & 0.113 & 0.872 & 1.477 & 1.817 & 1.353 \\
& & 0.8 & 0.710 & 1.781 & 0.999 & 15.690 & 1.039 & 0.887 & 15.706 & 2.062 & 1.336 \\
& 0.1 & 0.2 & 5.732 & 2.049 & 0.741 & 127.450 & 5.770 & 1.840 & 127.580 & 6.123 & 1.984 \\
& & 0.8 & -0.687 & 1.514 & 0.098 & 16.759 & 11.372 & 1.679 & 16.773 & 11.473 & 1.682 \\
\hline$\theta_{02}$ & 1 & 0.2 & 0.986 & 0.284 & 0.990 & 0.485 & 0.128 & 0.484 & 1.099 & 0.312 & 1.102 \\
& & 0.8 & 0.967 & 0.308 & 0.978 & 0.467 & 0.131 & 0.468 & 1.075 & 0.335 & 1.084 \\
& 0.5 & 0.2 & 0.901 & 0.211 & 0.958 & 1.191 & 0.162 & 1.036 & 1.494 & 0.266 & 1.411 \\
& & 0.8 & 1.356 & 0.795 & 1.009 & 18.897 & 16.040 & 1.050 & 18.946 & 16.060 & 1.456 \\
& 0.1 & 0.2 & -4.589 & -0.072 & 1.302 & 152.920 & 21.531 & 2.182 & 152.990 & 21.532 & 2.541 \\
& 0.8 & 2.991 & 4.000 & 2.074 & 19.761 & 130.070 & 1.976 & 19.986 & 130.130 & 2.865 \\
\hline
\end{tabular}

To summarize, the new estimator performs quite well in finite samples of small and moderate size uniformly over different levels of nonparametric identification. In contrast, IV and optimal IV estimates are rather sensitive to weak identification, which empirically confirms its uniform inconsistency. These simulations suggest that the new estimator can be a sensible and simple alternative to standard IV procedures when the researcher is concerned about weak or moderate identification.

\section{Estimating the Elasticity of Intertemporal Substitu- tion}

In its log-linearized version, the Consumption-based Capital Asset Pricing Model (CCAPM) studied in Hansen and Singleton (1982) leads to the conditional moment restriction

$$
E_{P}\left[\Delta c_{t+1}-\alpha-\psi r_{t+1} \mid Z_{t}\right]=0
$$

where $\psi$ is the elasticity of intertemporal substitution (EIS), $\Delta c_{t+1}$ is the growth rate of consumption, $r_{t+1}$ is the log gross return on some asset, $\alpha$ is a constant and $Z_{t}$ is a vector of variables in the agent's information set at time $t$. The parameters $\theta_{0}=(\alpha, \psi)^{\prime}$ can be estimated from (13) by several estimation strategies; see Hansen and Singleton (1983) and 
Hall (1988). We can estimate $\theta_{0}$ by a TSLS estimator with $\Delta c_{t+1}$ as the dependent variable; another is to apply TSLS with $r_{t+1}$ as the dependent variable; a third one is to use a method that is invariant to the normalization, such as Limited-Information Maximum Likelihood (LIML). Under strong IV identification, these methods should be asymptotically equivalent, so it should not matter which method to use. In practice, it has been shown that it greatly matters, which provides indirect evidence of weak instruments; see Stock and Wright (2000) and Neely et al. (2001). This empirical evidence has been extended to international data in Campbell (2003). The weak instruments problem may explain the apparently contradictory results that estimates of $\psi$ and $1 / \psi$ are simultaneously small and not statistically significant.

We revisit this issue using our new tools for an international data set considered in Campbell (2003) and Yogo (2004). ${ }^{7}$ The data consists of quarterly observations on equity markets at an aggregate level and macroeconomic variables for eleven countries: Australia (AUL), Canada (CAN), France (FR), Germany (GER), Italy (ITA), Japan (JAP), Netherlands (NTH), Sweden (SWD), Switzerland (SWR), the United Kingdom (UK) and the United States (USA). In addition, a longer time series is available at annual frequency for Sweden, the United Kingdom and the United States. The primary sources of international data are Morgan Stanley Capital International and the International Financial Statistics of the International Monetary Fund. The sample periods vary by country and frequency, see Table IV. A full description of the data is given in Campbell (2003). ${ }^{8}$

The asset returns used are the real interest rate, denoted by $r_{f, t}$, and the real aggregate stock return, denoted by $r_{e, t}$. The real stock return is constructed as log of the gross stock return deflated by the consumer price index. The real interest rate is constructed in the same way, using an available proxy for the short-term interest rate. Real consumption growth, denoted by $\Delta c_{t}$, is the first difference in log real consumption per capita. Following Yogo (2004), we use as instruments $Z_{t}=\left(r_{t-1}, \pi_{t-1}, \Delta c_{t-1}, d p_{t-1}\right)$, where $r_{t}$ is the nominal interest rate, $\pi_{t}$ is inflation, and $d p_{t}$ is the log dividend-price ratio.

Yogo (2004) provided formal evidence of weak instruments for these data sets and instruments. In particular, he showed that quite often the first-stage F-test was less than 5 in these regressions, see Table IV here, and he used inference methods (confidence intervals and test statistics) that were robust to identification failure. His main conclusion was that the EIS is small and not significant across the eleven countries considered.

\footnotetext{
${ }^{7}$ The data set is available at Motohiro Yogo's web page. We thank Motohiro for making the data available.

${ }^{8}$ This application involves time series, while the theory was presented for iid data. It is however straightforward to extend our uniform consistency results to time series. All we need is to replace the law of large numbers for iid data by the Ergodic Theorem. Extensions are also possible for uniform asymptotic normality under martingale difference errors, which arise naturally in applications such as the present Euler equation. In these extensions $P$ is still the (stationary) marginal distribution of $W_{t}$.
} 
The weak identification of IV comes here at not surprise, as is well-known that both consumption growth and asset returns are notoriously difficult to be linearly predicted. However, as this paper emphasizes, it is possible that these variables are linearly unpredictable but nonlinearly predictable using the set of instruments $Z_{t}$; see Guidolin et al. (2009) and references therein for extensive empirical evidence of this nonlinearity. We first investigate empirically the possibility of nonparametric identification in the next section.

\subsection{Testing for the Nonparametric Relevance Condition}

Our theoretical results (cf. Proposition 1) imply that the parameter $\theta_{0}=(\alpha, \psi)^{\prime}$ is identified in the regression (13) if and only if $Z_{t}$ is nonparametrically significant in the regression $E_{P}\left[r_{t+1} \mid Z_{t}\right]$, i.e.

$$
P\left(E_{P}\left[r_{t+1} \mid Z_{t}\right] \neq E_{P}\left[r_{t+1}\right]\right)>0 .
$$

Likewise, if $\psi \neq 0$, then $1 / \psi$ is identified from

$$
E_{P}\left[r_{t+1}-\beta-(1 / \psi) \Delta c_{t+1} \mid Z_{t}\right]=0
$$

provided

$$
P\left(E_{P}\left[\Delta c_{t+1} \mid Z_{t}\right] \neq E_{P}\left[\Delta c_{t+1}\right]\right)>0 \text {. }
$$

There is an extensive literature in econometrics on nonparametric tests for the hypothesis in (14) and (16). See, e.g., Bierens (1982) and Stinchcombe and White (1998). Here, we follow Bierens (1982) and use a Cramer-von Mises (CvM) test for the null hypothesis $H_{0}: P\left(E_{P}\left[Y_{t+1} \mid Z_{t}\right]=E_{P}\left[Y_{t+1}\right]\right)=1$ against $H_{1}: P\left(E_{P}\left[Y_{t+1} \mid Z_{t}\right] \neq E_{P}\left[Y_{t+1}\right]\right)>0$, where $Y_{t+1}$ denotes either $\Delta c_{t+1}, r_{f, t+1}$ or $r_{e, t+1}$, thus testing the nonparametric relevance conditions in (14) and (16). The test statistics are quadratic forms in the residuals $\hat{u}=\left(\hat{u}_{1}, \ldots, \hat{u}_{n}\right)^{\prime}$, with $\hat{u}_{t}=\left(Y_{t+1}-\bar{Y}_{n}\right)$ and $\bar{Y}_{n}=n^{-1} \sum_{t=1}^{n} Y_{t+1}$, computed as

$$
C v M_{n}=\frac{\hat{u}^{\prime} \Omega \hat{u}}{n \widehat{\sigma}^{2}}
$$

where $\Omega$ is defined after $(3)$ and $\widehat{\sigma}^{2}=n^{-1} \sum_{t=1}^{n} \hat{u}_{t}^{2}$. We standardize the components of $Z_{t}$ by their sample standard deviation, so tests become scale invariant. Note that one may think of $C v M_{n}$ as a nonparametric extension of the classical first-stage $F$-test in IV regression. ${ }^{9}$

The asymptotic distribution of $C v M_{n}$ is not pivotal, but its critical values can be approximated by a wild-bootstrap procedure; see Dominguez and Lobato (2003). That is, we

\footnotetext{
${ }^{9}$ The $F$-test is computed as $F=\left(\hat{u}^{\prime} P_{Z, n} \hat{u}\right) / n \widehat{\sigma}_{F}^{2}$, where $P_{Z, n}=Z\left(Z^{\prime} Z / n\right)^{-1} Z^{\prime}, \widehat{\sigma}_{F}^{2}=4 S S R_{u} /(n-5)$ and $S S R_{u}$ is the sum of unrestricted squared residuals.
} 
can approximate the asymptotic distribution of $C v M_{n}$ by that of

$$
C v M_{n}^{*}=\frac{\hat{u}^{* \prime} \Omega \hat{u}^{*}}{n \widehat{\sigma}^{* 2}}
$$

where $\hat{u}^{*}=\left(\hat{u}_{1}^{*}, \ldots, \hat{u}_{n}^{*}\right)^{\prime}, \hat{u}_{t}^{*}=\left(V_{t} \hat{u}_{t}-c_{n}\right), c_{n}=n^{-1} \sum_{t=1}^{n} V_{t} \hat{u}_{t}, \widehat{\sigma}^{* 2}=n^{-1} \sum_{t=1}^{n} \hat{u}_{t}^{* 2}$, and where $\left\{V_{t}\right\}_{t=1}^{n}$ is a sequence of $i i d$ random variables with zero mean, unit variance, bounded support and also independent of the sequence $\left\{Y_{t}, X_{t}, Z_{t}\right\}_{t=1}^{n+1}$. Examples of $\left\{V_{t}\right\}$ sequences are iid Bernoulli variables with

$$
P\left(V_{t}=0.5(1-\sqrt{5})\right)=b \quad P\left(V_{t}=0.5(1+\sqrt{5})\right)=1-b,
$$

where $b=(1+\sqrt{5}) / 2 \sqrt{5}$, or $P\left(V_{t}=1\right)=0.5$ and $P\left(V_{t}=-1\right)=0.5$. The critical values of $C v M_{n}^{*}$ are approximated by Monte Carlo simulations. Thus, the null hypothesis of lack of nonparametric relevance will be rejected at the $100 \alpha \%$ of significance when $C v M_{n} \geq c_{n, \alpha}^{*}$, where $c_{n, \alpha}^{*}$ is the $(1-\alpha)-t h$ empirical quantile of $B$ realizations of $C v M_{n}^{*}$. Alternatively, we can use the bootstrap $p$-values, $p_{n}^{*}$ say, rejecting $H_{0}$ when $p_{n}^{*}<\alpha$, where $p_{n}^{*}=\operatorname{Pr}\left(C v M_{n}^{*} \geq C v M_{n} \mid\left\{Y_{t}, X_{t}, Z_{t}\right\}_{t=1}^{n+1}\right)$.

Table IV reports the bootstrap p-values for the data sets considered and the three variables $\Delta c_{t+1}, r_{f, t+1}$ and $r_{e, t+1}$. The number of bootstrap replications is $B=5000$. For completeness we also report the values of the first-stage F test from Yogo (2004). This is useful in order to compare parametric and nonparametric identification failures. The F tests suggest low linear predictability for all countries and both frequencies in consumption growth and stock returns, but high predictability of interest rates. When looking for nonlinear (nonparametric) predictability we find that interest rates are highly predictable, stock returns are not predictable, and consumption growth shows low or no predictability for all countries but for UK and USA. Hence, two conclusions arise from our identification analysis: (i) the evidence of weak linear identification in international data found in Yogo (2004) and others can be extended to weak nonparametric identification when considering stock returns, and (ii) when using interest rates, it appears that $\psi$ and $1 / \psi$ are nonparametrically identified for UK and USA data, but $1 / \psi$ is weakly identified by IV methods. Thus, the UK and USA quarterly data highlight the difference between linear and nonlinear predictability, and hence the difference between lack of identification by linear methods and our nonparametric relevance assumption. In view of this evidence, in what follows we restrict our attention to these two countries and interest rates data. It is interesting to note that our findings agree with the extensive empirical evidence in Guidolin et al. (2009). These authors investigate nonlinear predictability in G7 countries and conclude that: "US and UK data return 
data appear to be "special," in the sense that good predictive performance demands the estimation of non-linear models".

TABLE IV

\begin{tabular}{ccccc}
\multicolumn{5}{c}{ Nonparametric Relevance Tests. Bootstrap P-values } \\
\hline \hline Country & Sample Period & $Y_{t+1}=\Delta c_{t+1}$ & $Y_{t+1}=r_{f, t+1}$ & $Y_{t+1}=r_{e, t+1}$ \\
\hline AUL & $1970.3-1998.4$ & $0.350(1.79)$ & $0.000(21.81)$ & $0.345(1.82)$ \\
CAN & $1970.3-1999.1$ & $0.446(3.03)$ & $0.000(15.37)$ & $0.599(2.51)$ \\
FR & $1970.3-1998.3$ & $0.901(0.17)$ & $0.000(38.43)$ & $0.614(3.09)$ \\
GER & $1979.1-1998.3$ & $0.541(0.83)$ & $0.005(17.66)$ & $0.820(0.69)$ \\
ITA & $1971.4-1998.1$ & $0.678(0.73)$ & $0.000(19.01)$ & $0.819(1.10)$ \\
JAP & $1970.3-1998.4$ & $0.501(1.18)$ & $0.000(8.64)$ & $0.344(3.49)$ \\
NTH & $1977.3-1998.2$ & $0.599(0.89)$ & $0.000(12.05)$ & $0.946(0.73)$ \\
SWD & $1970.3-1999.2$ & $0.622(0.48)$ & $0.000(17.08)$ & $0.151(2.24)$ \\
SWT & $1976.2-1998.4$ & $0.832(0.97)$ & $0.005(8.55)$ & $0.975(0.11)$ \\
UK & $1970.3-1999.1$ & $0.075(2.52)$ & $0.003(17.04)$ & $0.600(2.62)$ \\
USA & $1947.3-1998.4$ & $0.003(2.93)$ & $0.000(15.53)$ & $0.196(2.88)$ \\
\hline SWD & $1921-1994$ & $0.805(1.02)$ & $0.000(5.50)$ & $0.439(1.67)$ \\
UK & $1921-1994$ & $0.407(1.93)$ & $0.001(4.87)$ & $0.009(4.18)$ \\
USA & $1891-1995$ & $0.959(1.55)$ & $0.058(2.87)$ & $0.439(1.00)$ \\
\hline
\end{tabular}

Note: First stage F-statistics in parenthesis. Bootstrap replications $B=5000$.

\subsection{Uniformly Consistent Estimation and Inference}

We now proceed to estimate $\psi$ in (13) and $1 / \psi$ in (15) using our IIV estimator and interest rates and consumption growth for UK and USA data. For completeness we also report results for annual data. We compare in Table V our IIV estimator with Yogo's TSLS and LIML estimates. Standard errors, computed under the assumption of martingale difference errors, are provided in parenthesis. We also computed HAC standard errors with automatic lag-length choice, but results were very similar, so they are not reported. The first fact to note is that the three estimators give very different results, which may be considered as evidence of identification problems. Our IIV estimates for $\psi$ with quarterly data are considerably larger than those of the TSLS and LIML estimates, and viceversa for annual data. The case of quarterly UK data is particularly illuminating and highlights the benefits of the present approach. This is an example where there is weak identification by IV methods, 
but the parameters are nonparametrically identified by exploiting nonlinear (nonparametric) dependence between interest rates and instruments. Our estimate of the EIS for quarterly UK data is 0.5 , and is significantly different from zero. The TSLS estimate is much smaller (0.17) and not significant at 5\%. In contrast to the puzzling results with TSLS, we do not get contradictory results when estimating $1 / \psi$, with an estimate of 1.94 that is fairly consistent with the estimate of $\psi$. For USA quarterly data, we obtain an estimate of 0.66 , that although larger than the TSLS and LIML estimates, it is not significantly different from zero. Estimates of the EIS with annual data are small and no significantly different from zero, in agrement with previous studies.

\section{TABLE V}

Estimates: Interest Rates and Consumption Regressions

\begin{tabular}{cccccccc}
\hline \hline Country & Sample Period & \multicolumn{3}{c}{$\psi$} & & \multicolumn{3}{c}{$1 / \psi$} \\
\hline \multirow{3}{*}{ UK } & \multirow{2}{*}{$1970.3-1999.1$} & IIV & TSLS & LIML & IIV & TSLS & LIML \\
\cline { 3 - 8 } & & 0.50 & 0.17 & 0.16 & 1.94 & 1.06 & 6.21 \\
\multirow{2}{*}{ USA } & $1947.3-1998.4$ & 0.66 & 0.06 & 0.03 & 1.41 & 0.68 & 34.11 \\
& & $(0.49)$ & $(0.09)$ & $(0.10)$ & $(1.02)$ & $(0.48)$ & $(112.50)$ \\
\hline \multirow{2}{*}{ UK } & \multirow{2}{*}{$1921-1994$} & 0.08 & 0.26 & 0.28 & 5.57 & 2.40 & 3.52 \\
& & $(0.20)$ & $(0.12)$ & $(0.13)$ & $(4.85)$ & $(1.01)$ & $(1.65)$ \\
USA & \multirow{2}{*}{$1891-1995$} & -0.28 & -0.03 & -0.03 & -2.97 & -0.38 & -39.71 \\
& & $(0.34)$ & $(0.11)$ & $(0.16)$ & $(3.51)$ & 1.12 & $(257.54)$ \\
\hline
\end{tabular}

We now compare $95 \%$ asymptotic confidence intervals for the EIS based on the IIV estimates with those from other methods that are robust to weak identification. More concretely, we compare with confidence intervals computed inverting the Anderson-Rubin's test (AR, see Anderson and Rubin, 1949) and the conditional likelihood ratio (CLR) test of Moreira (2003). An excellent description of these procedures can be found in Yogo (2004). We first note that the confidence intervals are different across the different methods. This can be partly accounted for by the information provided by the nonlinearity in the data. For quarterly UK data, IIV provides confidence intervals consistent with larger values for the EIS than those provided by IV robust methods. In particular, according to the CLR the EIS is not significantly different from zero, in stark contrast with our finding using IIV estimates. Our confidence intervals suggest that for USA data the EIS is not significantly different from zero at 5\%. Moreover, it appears that there are serious identification problems with linear methods in the annual USA data as evidenced by the uninformative confidence intervals 
of the CLR test. On the contrary, our results indicate that the parameter $\psi$ is identified, although takes small values.

TABLE VI

\begin{tabular}{ccccc}
\multicolumn{5}{c}{ Confidence Intervals. Interest Rates } \\
\hline \hline Country & Sample Period & \multicolumn{3}{c}{$\psi$} \\
\hline & & IIV & AR & CLR \\
\cline { 3 - 5 } UK & $1970.3-1999.1$ & {$[0.10,0.90]$} & {$[0.04,0.28]$} & {$[-0.12,0.43]$} \\
USA & $1947.3-1998.4$ & {$[-0.31,1.63]$} & $\varnothing$ & {$[-0.19,0.22]$} \\
\hline UK & $1921-1994$ & {$[-0.32,0.49]$} & {$[-0.05,0.88]$} & {$[0.01,0.70]$} \\
USA & $1891-1995$ & {$[-0.94,0.38]$} & {$[-0.49,0.46]$} & {$[-\infty, \infty]$} \\
\hline
\end{tabular}

\subsection{Nonparametric Tests for Overidentifying Restrictions}

The proposed IIV estimator relies on the strict exogeneity assumption in (13). Much as in the classical linear case, we can tests for nonparametric overidentifying restrictions. This robustness checks provide some empirical validity to the previous results. To that end, we extend the nonparametric tests of Dominguez and Lobato (2003) to a linear regression setting (these authors consider the case of no covariates in the regression). The test statistics are of CvM type for testing $H_{0}: P\left(E_{P}\left[\Delta c_{t+1}-\alpha-\psi r_{f, t+1} \mid Z_{t}\right]=0\right)=1$, against the general nonparametric alternative $H_{1}: P\left(E_{P}\left[\Delta c_{t+1}-\alpha-\psi r_{t+1} \mid Z_{t}\right] \neq 0\right)>0$. The CvM test is computed as a quadratic form in the residuals $\hat{u}=\left(\hat{u}_{1}, \ldots, \hat{u}_{n}\right)^{\prime}$, with $\hat{u}_{t}=\Delta c_{t+1}-\widehat{\alpha}-\widehat{\psi} r_{f, t+1}$, $\hat{\theta}_{I I V}=(\widehat{\alpha}, \widehat{\psi})^{\prime}$, simply as

$$
C v M_{n}=\frac{1}{\widehat{\sigma}^{2} n^{2}} \hat{u}^{\prime} \Omega_{i n d}^{\prime} \Omega_{i n d} \hat{u},
$$

where $\Omega_{\text {ind }}=H \Psi, H=I_{n}-\mathbf{X}\left(\mathbf{X}^{\prime} \mathbf{X}\right)^{-1} \mathbf{X}^{\prime}, I_{n}$ is the $n \times n$ identity matrix, and $\Psi$ is the $n \times n$ matrix with elements $w_{t s}=1\left(Z_{t} \leq Z_{s}\right)$. The asymptotic distribution of $C v M_{n}$ is approximated by that of the bootstrap analogue $C v M_{n}^{*}=n^{-2} \hat{u}^{* \prime} \Omega_{i n d}^{\prime} \Omega_{i n d} \hat{u}^{*}$, where $\hat{u}^{*}=$ $\left(V_{1} \hat{u}_{1}, \ldots, V_{n} \hat{u}_{n}\right)^{\prime}$, and $\left\{V_{t}\right\}_{t=1}^{n}$ are generated from (17). This bootstrap can be justified along the lines of Dominguez and Lobato (2003). These tests require consistent estimation of the parameter $\theta_{0}$, which is supported by the empirical evidence above. The conclusion from applying these consistent strict exogeneity tests is that the strict exogeneity assumption is not rejected for all data sets. The bootstrap p-values for quarterly data are 0.252 and 0.282 for UK and USA, respectively. For annual data the bootstrap p-values are 0.146 and 0.703 , respectively. Hence, these tests validate previous inferences.

To sum, this application has shown that the new IIV estimator can complement existing inferential procedures under weak instruments in applications of economic interest. In addition, we have shown that is possible to test for the nonparametric relevance condition by 
means of consistent tests of conditional moment restrictions. The application to quarterly UK data provides an example where the EIS is weakly identified by linear methods, but strongly identified with our nonparametric methods.

\section{Conclusions and Extensions}

In this paper we have emphasized minimal conditions for identification of linear regression models when a strict exogeneity assumption holds. Under the minimal identifying assumption the commonly used IV estimators are not uniformly consistent, given the plausibility of weak instruments. We have proposed an estimator that is uniformly consistent under nearly the minimal identifying condition. The new estimator should be appealing to practitioners given its simplicity and robustness.

Optimal IV estimators, optimal in the sense of minimum asymptotic variance for regular estimators, also identify under the minimal identification assumption in linear models, but as shown in our simulations they are very sensitive to the level of nonparametric identification. This empirical evidence is theoretically supported by the lack of uniform consistency of optimal IV. The IIV estimator seems to outperform the optimal IV estimator for moderate and small sample sizes when nonparametric identification is weak. These results suggest that classical definitions of optimality in semiparametric models need to be challenged when optimal procedures are not uniformly valid.

It remains a topic of future research to compare our IIV estimator with other estimates such as the Fuller- $k$ class; see Section 6 in Stock et al. (2002) for a survey. Note that, unlike the new estimator, existing robust procedures do not identify the parameters under nearly minimal conditions. Whether these theoretical results translate into finite sample performance will be investigated in future work.

Another interesting extension is to testing for the nonparametric relevance condition in linear models with multivariate regressors. The negation of Assumption NR leads to a conditional moment restriction, which suggests that this assumption could be tested using existing consistent tests of conditional moment restrictions. There is, however, no guarantee that parameters in the resulting conditional moment restriction are identified, which invalidates the application of standard consistent tests. This issue is an interesting topic for future research.

More broadly, this paper has emphasized uniform consistent estimation under nearly minimal identifying conditions. Minimum distance estimators, as discussed by Wolfowitz (1957) and others, are good candidates for uniform consistent estimators. Developing such uniform procedures in general econometric models deserves further investigation. 


\section{Appendix: Mathematical Proofs}

Proof of Proposition 3: We shall prove that the negation of Assumption NR is equivalent to the negation of (7). That is, the negation of Assumption NR implies that there exists a non-zero vector, say $\alpha \in \mathbb{R}^{p}$, such that $\alpha^{\prime} E_{P}\left[m(Z) m^{\prime}(Z)\right] \alpha=0$, that is,

$$
E_{P}\left[\alpha^{\prime} X \mid Z\right]=0 \text { a.s. }
$$

Now, by the law of iterated expectations and Theorem 1 in Bierens (1982), (18) is equivalent to

$$
\alpha^{\prime} h_{x, P}(v)=E_{P}\left[E_{P}\left[\alpha^{\prime} X \mid Z\right] \exp \left(i v^{\prime} Z\right)\right]=0 \text { almost everywhere (a.e.) }
$$

Hence, $\alpha^{\prime} E_{V}\left[h_{x, P}(V) h_{x, P}^{c}(V)\right] \alpha=0$.

Proof of Theorem 1: Define the class of functions

$$
\mathcal{F}_{x}=\left\{w=(y, x, z) \rightarrow x \exp \left(i v^{\prime} z\right), v \in \Pi\right\},
$$

where $\Pi$ is a compact, convex subset of $\mathbb{R}^{k}$ with nonempty interior. Note that $\left|x \exp \left(i v^{\prime} z\right)\right| \leq$ $|x|$ and therefore $F_{x}(w)=|x|$ is an envelop for the class $\mathcal{F}_{x}$. We shall use a number of results from van der Vaart and Wellner (1996), which is hereinafter denoted by VW to save space.

Fix an arbitrary $\delta>0$, and choose a compact and convex set $\Pi \equiv \Pi_{\delta}$ such that

$$
\left|\int_{\mathbb{R}^{k} \backslash \Pi} \phi(v) d v\right| \leq \delta .
$$

Then, write

$$
\begin{aligned}
\left\langle h_{x_{j}, P_{n}}, h_{x_{k}, P_{n}}\right\rangle & =\int_{\mathbb{R}^{k}} h_{x_{j}, P_{n}}(v) h_{x_{k}, P_{n}}^{c}(v) \phi(v) d v . \\
& =\int_{\Pi} h_{x_{j}, P_{n}}(v) h_{x_{k}, P_{n}}^{c}(v) \phi(v) d v+\int_{\mathbb{R}^{k} \backslash \Pi} h_{x_{j}, P_{n}}(v) h_{x_{k}, P_{n}}^{c}(v) \phi(v) d v . \\
& =: I_{1 n, P}+I_{2 n, P} .
\end{aligned}
$$

We deal with $I_{1 n, P}$ by showing that the class $\mathcal{F}_{x}$ is Glivenko-Cantelli uniformly in $P \in \mathcal{P}$, as defined in VW (p. 167). To that end, we shall apply Theorem 2.8 .1 of that reference. First, 
note that the envelop satisfies

$$
\lim _{M \rightarrow \infty} \sup _{P \in \mathcal{P}} E_{P}[|X| 1(|X|>M)]=0
$$

by the uniformly bounded second moment of $X$ on $\mathcal{P}$.

Using standard empirical processes results in VW (p. 84 and Corollary 2.7.2) we conclude

$$
\sup _{Q} \log N\left(\varepsilon\left\|F_{x}\right\|_{Q, 1}, \mathcal{F}_{x},\|\cdot\|_{Q, 1}\right)=o(n)
$$

where the supremum in $Q$ is over the set of all discrete probability measures with atoms of size integer multiples of $1 / n, N\left(\varepsilon, \mathcal{F}_{x},\|\cdot\|_{Q, 1}\right)$ is the covering number defined in VW (p. 83), and $\|\cdot\|_{Q, 1}$ is the $L_{1}$ norm $\|F\|_{Q, 1}:=\int|F| d Q$. Then, we have by VW (Theorem 2.8.1) that $\mathcal{F}_{x}$ is Glivenko-Cantelli uniformly in $P \in \mathcal{P}$, and by the continuous mapping theorem

$$
I_{1 n, P}=\int_{\Pi} h_{x_{j}, P}(v) h_{x_{k}, P}^{c}(v) \phi(v) d v+o_{P}(1) \text { uniformly in } P \in \mathcal{P} \text {. }
$$

On the other hand, it is straightforward to show that

$$
I_{2 n, P}=O_{P}(\delta) \text { uniformly in } P \in \mathcal{P} \text {, }
$$

by (19) and the inequality $\left|h_{x_{j}, P_{n}}(v) h_{x_{k}, P_{n}}^{c}(v)\right| \leq\left|P_{n} F_{x}\right|^{2}=O_{P}(1)$ uniformly in $P \in \mathcal{P}$. Since $\delta>0$ was arbitrary, conclude from (20) that for all $j, k=1, \ldots, p$,

$$
\left\langle h_{x_{j}, P_{n}}, h_{x_{k}, P_{n}}\right\rangle=\left\langle h_{x_{j}, P}, h_{x_{k}, P}\right\rangle+o_{P}(1) \text { uniformly in } P \in \mathcal{P} \text {. }
$$

The proof of

$$
\left\langle h_{x_{j}, P_{n}}, h_{y, P_{n}}\right\rangle=\left\langle h_{x_{j}, P}, h_{y, P}\right\rangle+o_{P}(1) \text { uniformly in } P \in \mathcal{P}
$$

follows the same arguments, and hence is omitted. Together, this implies the statement of the Theorem.

Proof of Theorem 2: The arguments of the proof of Theorem 1 yield that $h_{x, P_{n}} \stackrel{L_{2}}{\longrightarrow}$ $h_{x}$ uniformly in $P \in \mathcal{P}$, and therefore, uniformly in $P \in \mathcal{P}^{A N} \subset \mathcal{P}$. On the other hand, by 
Chebyshev's inequality, for any $M>0$,

$$
\begin{aligned}
\sup _{P \in \mathcal{P}^{A N}} P\left(\left\|\sqrt{n} h_{\varepsilon, P_{n}}\right\|>M\right) & \leq M^{-2} \sup _{P \in \mathcal{P}^{A N}} E_{P}\left[\left\|\sqrt{n} h_{\varepsilon, P_{n}}\right\|^{2}\right] \\
& \leq M^{-2} \sup _{P \in \mathcal{P}^{A N}} E_{P}\left[\varepsilon^{2}\right] \\
& \leq M^{-2} C,
\end{aligned}
$$

where the second inequality uses the $i i d$ and strict exogeneity assumption. Thus, $\sqrt{n} h_{\varepsilon, P_{n}}=$ $O_{P}(1)$ uniformly in $P \in \mathcal{P}^{A N}$ in $L_{2}(\phi)$. These asymptotic results and the continuity of the inner product yield, uniformly in $P \in \mathcal{P}$, the expansion

$$
\sqrt{n}\left(\hat{\theta}_{I I V}-\theta_{0}\right)=\left\langle h_{x, P}, h_{x, P}\right\rangle^{-1} \sqrt{n}\left\langle h_{x, P}, h_{\varepsilon, P_{n}}\right\rangle+o_{P}(1) .
$$

Fix an arbitrary $\lambda \in \mathbb{R}^{p}$, with $|\lambda|=1$. Define the influence function

$$
\psi(W, P):=\lambda^{\prime} \Gamma_{P}^{-1 / 2}\left\langle h_{x, P}, h_{x, P}\right\rangle^{-1} \varepsilon H_{x, P}(Z),
$$

and note that

$$
E_{P}[\psi(W, P)]=0 \text { and } E_{P}\left[\psi^{2}(W, P)\right]=1 .
$$

Furthermore,

$$
\begin{aligned}
\sup _{P \in \mathcal{P}^{A N}} E_{P}\left[\psi^{2}(W, P) 1(\psi(W, P)>M)\right] & \leq C \sup _{P \in \mathcal{P}^{A N}} E_{P}[1(\psi(W, P)>M)] \\
& \rightarrow 0 \text { as } M \rightarrow \infty
\end{aligned}
$$

Therefore, by the uniform CLT of Petrov (1975, p. 118) the uniform asymptotic normality of $\sqrt{n}\left(\hat{\theta}_{I I V}-\theta_{0}\right)$ follows. 


\section{References}

[1] Amemiya, T. (1974): "The Non-linear Two-Stage Least Squares Estimator," Journal of Econometrics, 2, 105-110.

[2] Amemiya, T. (1977): "The Maximum Likelihood and the Non-linear Three-Stage Least Squares Estimator in the General Non-linear Simultaneous Equation Model," Econometrica, 45: 955-968.

[3] Anderson, T.W. and Rubin, H. (1949): "Estimation of the Parameters of a Single Equation in a Complete System of Stochastic Equations," Annals of Mathematical Statistics, 20, 46-63.

[4] Andrews, D.W.K and Guggenberger, P. (2009): "Validity of Subsampling and 'Plug-in Asymptotic' Inference for Parameters Defined by Moment Inequalities," Econometric Theory, 25(3): 669-709.

[5] Andrews, D.W.K. and Stock, J. (2005): "Inference with Weak Instruments," invited survey note for the 2005 World congress of the Econometric Society.

[6] Bahadur, R.R and Savage, L.J. (1956): "The Nonexistence of Certain Statistical Procedures in Nonparametric Problems," Annals of Mathematical Statistics, 27, 11151122.

[7] Belloni, A., Chernozhukov, V., Fernandez-Val, I. and C. Hansen (2015): "Program Evaluation with High-Dimensional Data," arXiv:1311.2645v5.

[8] Bickel, P.J., Klaassen, C.A., Ritov, Y. and Wellner, J.A. (1993): Efficient and Adaptive Estimation for Semiparametric Models. Johns Hopkins Univ. Press, Baltimore.

[9] Bierens, H. J. (1982): "Consistent Model Specification Tests," Journal of Econometrics, 20, 105-134.

[10] Cai, Z., Das, M., Xiong, H. and Wu, X. (2006): "Functional Coefficient Instrumental Variables Models," Journal of Econometrics, 133, 207-241.

[11] Campbell, J.Y. (2003): "Consumption-Based Asset Pricing", Chapter 13 in George Constantinides, Milton Harris, and Rene Stulz eds. Handbook of the Economics of Finance Vol. IB, North-Holland, Amsterdam, 803-887. 
[12] Carrasco, M. (2012): "A Regularization Approach to the Many Instruments Problem," Journal of Econometrics, 170, 383-398.

[13] Chamberlain, G. (1987): "Asymptotic Eficiency in Estimation with Conditional Moment Restrictions," Journal of Econometrics, 34, 305-334.

[14] DAs, M. (2005): "Instrumental Variables Estimators of Nonparametric Models with Discrete Endogenous Regressors," Journal of Econometrics, 124, 335-361.

[15] Domínguez, M. And Lobato, I. N. (2003): "A Consistent Test for the Martingale Difference Hypothesis," Econometric Reviews, 22, 351-377.

[16] Dominguez, M.A. And Lobato, I.N. (2004): "Consistent Estimation of Models Defined by Conditional Moment Restrictions," Econometrica, 72, 1601-1615.

[17] Dufour, J.M. (1997): "Some Impossibility Theorems in Econometrics with Applications to Structural and Dynamic Models," Econometrica, 65, 1365-1387.

[18] Escanciano, J. C. (2006): "A Consistent Diagnostic Test for Regression Models Using Projections," Econometric Theory, 22, 1030-1051.

[19] Guidolin, M. Hyde, S. McMillan, D. and Ono, S. (2009): "Non-linear Predictability in Stock and Bond Returns: When and Where is it Exploitable?," International Journal of Forecasting, 25, 373-399.

[20] Hall, R. E. (1988): "Intertemporal Substitution in Consumption," Journal of Political Economy, 96, 339-357.

[21] Hansen, L. P. And Singleton, K. J. (1982): "Generalized Instrumental Variables Estimation of Nonlinear Rational Expectations Models," Econometrica, 50, 1269-1286.

[22] Hansen, L. P. And Singleton, K. J. (1983): "Stochastic Consumption, Risk Aversion, and the Temporal Behavior of Asset Returns," Journal of Political Economy, 91, 249-265.

[23] Imbens, G. And Manski, C.F (2004): "Confidence Intervals for Partially Identified Parameters," Econometrica, 72, 1845-1857.

[24] KASY, M. (2015): "Uniformity and the Delta Method," unpublished manuscript.

[25] Leeb, H. And Pötscher, B. M. (2005): "Model selection and inference: Facts and fiction," Econometric Theory, 21, 21-59. 
[26] Linton, O. (2002): "Edgeworth Approximations for Semiparametric Instrumental Variable Estimators and Test Statistics", Journal of Econometrics, 106, 325-368.

[27] Manski, C.F. (1988): Analog Estimation Methods in Econometrics, London: Chapman and Hall.

[28] Mikusheva, A. (2007): "Uniform Inference in Autoregressive Models," Econometrica, $75,1411-1452$.

[29] Moreira, M.J. (2003): "A Conditional Likelihood Ratio Test for Structural Models," Econometrica, 71, 1027-1048.

[30] Neely, C., Roy, A. And Whiteman, C.H. (2001): "Risk Aversion versus Intertemporal Substitution: A Case Study of Identification Failure in the Intertemporal Consumption CAPM," Journal of Business and Economic Statistics, 19 395-403.

[31] NeWEy, W. (1990): "Efficient Instrumental Variables Estimation of Nonlinear Models," Econometrica 58, 809-837.

[32] Petrov, V. V. (1975): Sums of independent random variables. Springer, New York.

[33] Pötscher, B. M. (2002): "Lower Risk Bounds and Properties of Confidence Sets for Ill-Posed Estimation Problems with Applications to Spectral Density and Persistence Estimation, Unit Roots, and Estimation of Long Memory Parameters," Econometrica, 70, 1035-1065.

[34] Robinson, P. M. (1976): "Instrumental Variables Estimation of Differential Equations," Econometrica, 44, 765-776.

[35] Stinchcombe, M. and White, H, (1998): "Consistent Specification Testing with Nuisance Parameters Present only Under the Alternative," Econometric Theory, 14, 295-325.

[36] Staiger, D. And Stock, J.H. (1997): "Instrumental Variables Regression with Weak Instruments," Econometrica, 65, 557-586.

[37] Stock, J.H. And Wright, J.H. (2000): "GMM with Weak Identification," Econometrica, 68, 1055-1096.

[38] Stock, J. H., Wright, J. H. And Yogo, M. (2002): "A Survey of Weak Instruments and Weak Identification in Generalized Method of Moments," Journal of Business and Economic Statistics, 20, 518-529. 
[39] van der Vaart, A. W., and J. A. Wellner (1996): Weak Convergence and Empirical Processes with Applications to Statistics, Springer Series in Statistics. SpringerVerlag, New York, 1 edn.

[40] Wolfowitz, J. (1957): "The minimum distance method," The Annals of Mathematical Statistics, 28, 75-88.

[41] Wooldridge, J.M. (2002): Econometric Analysis of Cross Section and Panel Data. Cambridge, MA: MIT Press.

[42] Yogo, M. (2004): "Estimating the Elasticity of Intertemporal Substitution When Instruments Are Weak," Review of Economics and Statistics, 86, 797-810. 